\title{
Anticonvulsant Prophylaxis for Patients with Brain Tumours: Insights from Clinical Trials
}

Can. J. Neurol. Sci. 2003; 30: 89-90

Seizures are a common symptom of patients with intracranial neoplasms. Approximately 20 to $40 \%$ of patients with primary or metastatic brain tumours develop seizures during the course of their illness. ${ }^{1-3}$ Most seizures in patients with brain tumours are brief generalized or partial seizures, although a minority can result in status epilepticus or death. More significantly, seizures impact significantly on the quality of life of patients with brain tumours, causing injury and fear, and interfering with driving and work.

Although seizure frequency in patients with brain tumours and epilepsy can effectively be reduced by anticonvulsants, the use of these drugs to prevent an initial seizure in this patient population remains controversial. The issues surrounding the use of prophylactic anticonvulsants in patients with brain tumours are complex, and involve a cost/benefit analysis that is difficult to resolve. Anticonvulsants are expensive, associated with common and occasionally severe toxicities, require occasional monitoring of serum drug concentrations, and are inconvenient as evidenced by high rates of patient non-compliance.

The use of anticonvulsants in patients with brain tumours presents unique problems because anticonvulsants interfere with the metabolism of corticosteroids and a variety of chemotherapeutic agents, reducing their efficacy. Moreover, the ability of anticonvulsants to prevent an initial seizure in a patient with a brain tumour has not been demonstrated despite a number of retrospective reviews, and now at least two prospective randomised trials, including the study of Forsyth et al in this issue of the CJNS., ${ }^{4}$ The lack of evidence for the efficacy of prophylactic anticonvulsants for patients with brain tumours has prompted the American Academy of Neurology to issue recently a practice parameter recommending that prophylactic anticonvulsants not be used in this group of patients. ${ }^{6}$ Nonetheless, many patients with brain tumours are placed on prophylactic anticonvulsants by neurosurgeons, neurologists and oncologists who continue this practise presumably on the basis of personal bias.

In an attempt to determine whether anticonvulsants are effective as prophylactic agents in patients with a variety of brain tumours, Forsyth et $\mathrm{al}^{4}$ performed a multi-institutional randomised unblinded prospective trial of phenytoin in 100 patients with primary and metastatic brain tumours and no prior seizures. Patients were enrolled within one month of diagnosis, and stratified according to primary and metastatic brain tumours. Treated and observed cohorts were well-balanced with respect to significant baseline characteristics, including gender, performance status, and primary versus metastatic tumours. Patients who were placed on phenytoin had regular serum anticonvulsant levels measured to ensure therapeutic drug levels and monitor compliance. Frequent follow-up in both groups ensured vigilant recording of seizure frequency and drug toxicities. The trial was designed to detect a significant benefit from anticonvulsant prophylaxis: a reduction in absolute seizure frequency from a presumed $20 \%$ in the untreated cohort to $5 \%$ in those receiving phenytoin. To have a reasonable likelihood of detecting this difference, the trial planned on a total enrolment of 300 patients. The study was closed prematurely because seizure frequency and patient survival in both groups was less than expected, necessitating a revised target enrolment of 900 patients, a target that was correctly deemed unreasonable. Despite the early termination of this study, the key findings are interesting and valuable.

Most significantly, prophylactic phenytoin did not reduce the likelihood of a first seizure in patients with primary or metastatic brain tumours despite a median follow-up of almost six months. Furthermore, phenytoin did not alter seizure morphology in a way that could be considered beneficial; generalized seizures were equally common in both treated and observed groups. For unknown reasons, females were more likely to experience a seizure, but no other prognostic factors for seizures could be identified. Importantly, a first seizure was a common sign of tumor progression in this study, and this observation should prompt physicians to consider imaging any patient with a known brain tumour who develops new seizures or unexplained worsening of seizures.

Although patients on anticonvulsants claimed to be compliant with their medicines, serum drug levels indicated that only $53 \%$ were compliant. However, a therapeutic phenytoin serum level did not reduce the risk of a first seizure in the cohort assigned to prophylactic phenytoin, and most seizures could not be attributed to subtherapeutic drug levels. Finally, despite ongoing concerns surrounding the side-effects of anticonvulsants, this study did not record significant or frequent anticonvulsant toxicities, and few patients had to discontinue treatment and withdraw for this reason.

Although the study of Forsyth et al was closed prematurely, and thus could not definitively determine whether prophylactic anticonvulsants were effective in preventing the first seizure in patients with brain tumours, it does provide very useful clinical information. The results of this trial, in addition to other published studies including one where divalproex sodium was the prophylactic anticonvulsant used in a prospective and double-blinded placebo-controlled manner, ${ }^{5}$ should convince the clinician that anticonvulsants do not safeguard against an initial seizure in this vulnerable population. Consequently, clinicians generally should refrain from prescribing anticonvulsants for brain tumour patients who are seizure-free, and should consider 
tapering and eliminating anticonvulsants in patients who have been placed on anticonvulsants for prevention of an initial seizure.

The admitted and inherent limitations of the trial by Forsyth et al, and lingering questions surrounding the value, if any, of prophylactic anticonvulsants for patients with brain tumours may not convince clinicians to alter their behaviour of prescribing prophylactic anticonvulsants for specific subgroups of patients with brain tumours. For example, it is believed that patients with low-grade gliomas and those with metastases from cancers such as melanoma are at particularly high risk of seizures. ${ }^{7,8}$ Furthermore, anticonvulsants continue to be prescribed by neurosurgeons for patients with brain tumours in the belief that they prevent perioperative convulsions. It is conceivable that prophylactic anticonvulsants may be a reasonable strategy for selected high-risk patients, and for brief use surrounding neurosurgical intervention. It is also possible that anticonvulsants other than phenytoin or divalproex sodium might be effective prophylactic agents. However, to answer this question would require a randomised trial. The work of Forsyth et al is particularly instructive here; given this experience and the methodological challenges inherent in all such studies, it is unlikely that a trial powered to answer definitively the question of prophylactic anticonvulsants for selected patients with brain tumours will ever be conducted.

\section{REFERENCES}

1. Posner JB. Clinical manifestations of brain metastases. In: Gilbert HA, Posner JB, Weiss L, eds. Brain Metastases. Boston: GK Hall, 1980: 189-207.

2. Paillas JE, Pellet W. Brain metastases. In: Vinken PJ, Bruyn GW, eds. Handbook of Neurology. Amsterdam: North Holland 1975: 201-232.

3. Zimm S, Wampler GL, Stablein D, et al. Intracerebral metastases in solid-tumor patients: natural history and results of treatment. Cancer 1981; 48: 384-394.

4. Forsyth PA, Weaver S, Fulton D, et al. Difficulties in answering an important question in neuro-oncology: prophylactic anticonvulsants in patients with brain tumour. Can J Neurol Sci 2003; 30: 106-112.

5. Glantz MJ, Cole BF, Friedberg MH, et al. A randomised, blinded, placebo-controlled trial of divalproex sodium prophylaxis in adults with newly diagnosed brain tumours. Neurology 1996; 46:985-991.

6. Glantz MJ, Cole BF, Forsyth PA, et al. Practice parameter: anticonvulsant prophylaxis in patients with newly diagnosed brain tumors. Neurology 2000; 54: 1886-1893.

7. Bullard DE, Rawlings CE 3rd, Phillips B, et al. Oligodendroglioma: an analysis of the value of radiation therapy. Cancer 1987; 60: 2179-2188.

8. Byrne TN, Cascino TL, Posner JB. Brain metastases from melanoma. J Neuro-Onc 1983; 1: 313-317.

Warren P. Mason

Toronto, $\mathrm{ON}$ 